\title{
Propiedades Psicométricas de la Versión Española de la Abeer Children Dental Anxiety Scale (ACDAS) para la Medición de Ansiedad Dental en Niños
}

\author{
Psychometric Properties of the Spanish Version of the Abeer Children \\ Dental Anxiety Scale (ACDAS) to Dental Anxiety in Children
}

Ana Cristina Mafla'; Fredy Hernán Villalobos²; Wilson Marino Pinchao Ramírez ${ }^{3}$ \& Diego Fernando Lucero Yela ${ }^{3}$

MAFLA, A. C.; VILLALOBOS, F. H; PINCHAO, R. W. M. \& LUCERO, Y. D. F. Propiedades psicométricas de la versión española de la Abeer Children Dental Anxiety Scale (ACDAS) para la medición de ansiedad dental en niños. Int. J. Odontostomat., 11(2):182-191, 2017.

RESUMEN: El proceso de validez y fiabilidad de las escalas de ansiedad dental en español para niños ha sido escasa. El objetivo de esta investigación fue evaluar las propiedades psicométricas de la adaptación española de la Abeer Children Dental Anxiety Scale (ACDAS) como una medida de auto-reporte de ansiedad dental en niños. EI ACDAS incluye 13 ítems sobre las reacciones de ansiedad a estímulos de la atención odontológica y un componente cognitivo evaluado a los niños, padres y operadores. Se adaptó la ACDAS al idioma Español, luego se determinaron las propiedades psicométricas en una muestra de 200 pacientes niños de la Clínica Odontológica de la Universidad Cooperativa de Colombia, Pasto, Colombia. La validez estructural de la escala fue estimada con un Análisis Factorial Exploratorio (AFE) y su fiabilidad fue establecida a través de la consistencia interna ( $\alpha$ de Cronbach). La muestra consecutiva fue comprendida de 112 hombres y 88 mujeres y sus edades estuvieron entre 5 a 10 años. El AFE determinó una estructura de cuatro factores que representó el $72,12 \%$ de la varianza explicada. Se nombró a los factores de acuerdo con las reacciones de ansiedad a estímulos potencialmente nocivos como "estímulos pre-intervención", "estímulos restrictivos/invasivos", "estímulos inocuos (tacto, gusto)" y "estímulos inocuos (olfato, oído)". La consistencia interna de las sub-escalas fue 0,$88 ; 0,71 ; 0,84$ y 0,76 respectivamente. Las sub-escalas mostraron una buena convergencia, ser discriminantes y validez de constructo. Los resultados sugieren que la ACDAS es un instrumento válido y fiable, y clínicamente útil para la evaluación de la ansiedad dental en niños.

PALABRAS CLAVE: ansiedad dental, cognición, niños, encuesta y cuestionarios, psicometría.

\section{INTRODUCCIÓN}

La ansiedad es una respuesta multi-sistémica que aparece cuando el individuo cree que existe un peligro o amenaza, es decir, es subjetiva (lo que hace que varíe de una persona a otra) y genera un serio impacto en la vida cotidiana, convirtiéndose así en una barrera significativa para la demanda de atención en salud oral (Al-Namankany, et al., 2012a,b). La ansiedad dental puede definirse como un sentimiento exagerado de miedo originado por la visita al odontólogo como una reacción a un peligro que se espera. A diferencia de la medicina, nuestra profesión, se caracteriza porque en la consulta odontológica normalmente se realizan procedimientos de menor a mayor complejidad, y esto es identificado por las personas y pacientes. Aunque en menor grado, este malestar podría ser comparable con la ansiedad pre-operatoria, muy frecuente en el área médica (Norris \& Baird, 1967). En general, un gran número de niños tienen temor a los procedimientos odontológicos. Por ejemplo, en edades de 6-12 años

\footnotetext{
${ }^{1}$ Odontóloga, Máster en Salud Pública, Profesora Asistente, Facultad de Odontología, Universidad Cooperativa de Colombia, Pasto, Colombia. ${ }^{2}$ Psicólogo, PhD en Psicología Clínica y de la Salud, Profesor Asociado Departamento de Psicología, Universidad de Nariño, Pasto, Colombia.

${ }^{3}$ Estudiante de Odontología, Facultad de Odontología, Universidad Cooperativa de Colombia, Pasto, Colombia.
} 
en Rumania la prevalencia de ansiedad dental fue 22,68 \% (Popescu et al., 2014), y en Irán de 29,33\% (Paryab \& Hosseinbor, 2013).

La ansiedad dental puede complicar la aplicación de tratamientos odontológicos (Arapostathis, et al., 2008) conduciendo al progreso de enfermedades orales que originan la pérdida dental, por lo cual es importante que los odontólogos identifiquen a los niños con estos temores. A través de los años, se han desarrollado diferentes escalas con el objetivo de medir esta condición en este grupo, es así, que en 1979 se publica el Venham Picture Test - VPT (Venham, 1979; Venham \& Gaulin-Kremer, 1979), en 1982 el Children's Fear Survey Schedule-Dental Subscale (CFSS-DS) (Cuthbert \& Melamed, 1982), y veinte años más tarde, el Facial Image Scale (FIS) (Buchanan \& Niven, 2002).

El Venham Picture Test (VPT) fue elaborado porque no existían instrumentos para niños de menor edad que midieran ansiedad en situaciones estresantes, en donde su respuesta no fuera verbal. Este instrumento utiliza figuras de niños con diferentes estados emocionales como estar feliz, asustado, llorando, triste, con rabia y miedo a quedarse quieto. La versión final consistió en 8 figuras de niños basada en las correlaciones inter-test e impresiones clínicas. Se aplicó en niños de 3-8 años y obtuvo un $\alpha$ de Cronbach de 0,83 lo que indicó un alto grado de consistencia interna (Venham ; Venham \& GaulinKremer).

Más adelante, se diseñó el Children's Fear Survey Schedule-Dental Subscale (CFSS-DS), que consta de 15 items relacionados con estímulos como inyecciones o tener la boca abierta, entre otros. La puntuación total oscila entre 15 y 75 , los valores más altos indican mayor miedo a los procedimientos odontológicos. Una versión griega mostró una buena consistencia interna con un $\alpha$ de Cronbach de 0,85 (Arapostathis, et al.) y una croata de 0,83 (Majstorovic, et al., 2003).

Por su parte, la Facial Image Scale (FIS) es otro instrumento utilizado para evaluar ansiedad, a partir de los 3 años, la cual consiste en el uso de cinco figuras de la cara que muestran características afectivas que van desde una extrema actitud negativa hasta una positiva, con valores de 1 a 5 . Las pruebas de fiabilidad muestran un buen acuerdo con el índice de Kappa entre la auto-evaluación de los niños con la de sus padres $(n=50, K a p p a=$
0,66; $\mathrm{P}<0,001)$ y en menor grado con la de los odontólogos ( $n=50 ;$ Kappa $=0,45 ; P<0,05)$ cuando el niño estaba en la silla odontológica (Buchanan \& Niven, 2003). Ha sido muy útil cuando se ha medido de manera secuencial los niveles de ansiedad en niños de 6 a 7 años después de varias visitas al odontólogo (de Menezes Abreu et al., 2011).

La Escala Abeer (Al-Namankany, et al., 2012a,b), consta de dos partes, la primera tiene 13 preguntas que miden la ansiedad percibida por los niños ante diferentes procedimientos odontológicos y la segunda (cognitiva) se centra en valoraciones que hacen niño, padre y operador. Después de analizar algunas escalas de ansiedad dental en niños, observamos que no existen muchos métodos de medición, existen limitaciones en sus propiedades psicométricas, o que no han sido suficientemente validados en contextos latinoamericanos. Por esta razón, el objetivo de este estudio es determinar las propiedades psicométricas de una versión en español de la Escala Abeer para la medición de ansiedad dental en niños colombianos.

\section{MATERIAL Y MÉTODO}

Se diseñó un estudio observacional, analítico, de corte transversal. Para esta investigación se llevó a cabo un muestreo por casos consecutivos, en el que se evaluaron 200 pacientes pediátricos que asistieron a la Clínica Odontológica Integral de Niños de la Universidad Cooperativa de Colombia de Pasto de Julio a Noviembre de 2015. Se incluyó a niños y niñas de 5 a 10 años, con experiencias previas odontológicas y que sus padres aceptaran que su hijo voluntariamente participara en el estudio. Se excluyeron a niños de difícil manejo o poco colaboradores, con enfermedades físicas o mentales que no les permitieran responder el cuestionario.

Procedimiento. Para el desarrollo del estudio se llevaron a cabo los siguientes pasos: 1). Traducción y adaptación de la Escala Abeer (Al-Namankany, et al., 2012a,b). La escala fue revisada y se realizó una traducción directa e inversa al idioma Español, por dos profesionales quienes tenían suficiencia en el idioma inglés y en el tema específico (psicólogo y odontólogo). De acuerdo con aspectos culturales, nivel económico y desarrollo tecnológico, se modificó la pregunta 12 "Wearing a small rubbery mask on your nose to breathe special gas to help you feel 
comfortable during treatment?" (Ponerse una máscara de plástico en su nariz para respirar un gas especial que le ayude a sentirse cómodo durante el tratamiento?) por la pregunta "Usar una tela de caucho en tu boca durante el tratamiento?", y la pregunta 13 "Having a pinch feeling on the back of your hand? (Tener la sensación de un pellizco en el dorso de la mano?"), por "Ser sujetado (a) las manos y brazos?" 2). Se diseñó un formato de recolección de datos que incluía la Escala Abeer adaptada al idioma Español, que es evaluada con una modificación del Facial Image Scale, con tres opciones, también se registraron variables como edad y sexo (Fig. 1). 3). Se realizó una calibración a los investigadores para la entrevista a los niños. 4). Se organizó los horarios y el lugar donde se recolectaría la información. 5.) Se solicitó el consentimiento a los padres para que los niños respondan la encuesta.

Análisis Estadístico. Se realizó un análisis factorial exploratorio para conocer la estructura de la escala, se calculó la prueba de esfericidad de Bartlett y la prueba de adecuación de la muestra Kaiser-Meyer-Olkin (KMO). La extracción de factores se realizó mediante el método de componentes principales. Se calcularon las comunalidades, autovalores y el porcentaje de varianza explicada. Se estimó la correlación de Spearman de cada ítem con la puntuación total. La consistencia interna se evaluó mediante el $\alpha$ de Cronbach. Para la comparación de la escala del operador y la edad con las sub-escalas, se empleó la prueba estadística ANOVA. Se tomaron los valores del Índice de Kappa de Cohen, para determinar el acierto entre los reportes del operador y los auto-informes de los niños, de acuerdo a la ansiedad observada y percibida. Además, se calcularon los valores $Z$, para determinar la prevalencia de ansiedad en el grupo de niños en donde los valores mayores a 1 representaban presencia de ansiedad. La organización de los resultados se realizó en figuras y tablas. Los datos se procesaron en el paquete estadístico IBM SPSS (International Business Machines Corporation, Statistical Package for Social Science v. 23, Armok, NY, USA).

\section{RESULTADOS}

De los 250 niños que asistieron a las Clínicas de Odontopediatría en el II período de 2015, 200 fueron entrevistados para una tasa de respuesta del 80 $\%$. Del grupo evaluado 112 (56 \%) de los participantes fueron hombres y 88 (44\%) fueron mujeres, entre 5 y 10 años, con una media de 7,46 años (DE $=1,34$ ). Los niños pertenecían a estratos 1 y 2 del municipio de Pasto.

Aceptación del cuestionario. Todos los niños, padres y estudiantes de odontología contestaron las preguntas de la encuesta en el estudio, lo que supone una tasa de aceptabilidad general del $100 \%$.

Validez de la estructura interna. El índice de adecuación de KMO fue 0,847 y la prueba de esfericidad de Bartlett ( chi $\left.^{2}=1254,24 ; \mathrm{gl}=78 ; \mathrm{P}<0,001\right)$ mostraron que los ítems estaban inter-correlacionados entre sí y que los datos cumplían los requisitos para la realización del análisis factorial. Las comunalidades encontradas oscilaron entre 0,57 y 0,81 . Tanto el gráfico de sedimentación (Fig. 2) como la tabla de varianza explicada con un valor de $72,11 \%$, demostraron una estructura de 4 factores (Tabla I). Se llevó a cabo una rotación oblicua Oblimin (delta=0), pues se partió del supuesto que puede haber una correlación entre los componentes de la escala. Al revisar la matriz de patrón se observó que todos los ítems expresaron saturaciones elevadas que oscilaban entre 0,878 y 0,622 en sus respectivos componentes (Tabla II).

El primer factor con saturaciones entre 0,878 y 0,664, se denominó ansiedad ante la «pre-intervención», ya que agrupa los ítems relacionados con actividades previas y que no generan peligro, ni riesgo, como sentarse en la sala de espera, acostarse en la silla odontológica, ser revisado con un espejo o ser atendido por el profesional que use tapabocas. Sin embargo, son situaciones que generan ansiedad, ya que son anticipatorias a lo desconocido y probablemente se asocian a la posibilidad futura de

Tabla I. Varianza explicada por los componentes de la Escala de Ansiedad Dental Abeer para niños adaptada.

\begin{tabular}{cccc}
\hline \multirow{2}{*}{ Componente } & \multicolumn{3}{c}{ Sumas de extracción de cargas al cuadrado } \\
\cline { 2 - 4 } & Total & \% de varianza & \% acumulado \\
\hline 1 & 5,392 & 41,474 & 41,474 \\
2 & 1,837 & 14,129 & 55,603 \\
3 & 1,142 & 8,782 & 64,386 \\
4 & 1,005 & 7,734 & 72,119 \\
\hline
\end{tabular}




\section{A. Auto-reporte del niño}

Me gustaría que por favor me digas que tan asustado (a) o relajado (a) se siente con el odontólogo. Por favor usa la siguiente escala de 1 a 3 y marca $(X)$ debajo de la cara de cómo te sientes ahora:

\section{CÓMO TE SIENTES ACERCA DE:}

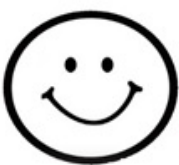

1. Estoy Feliz
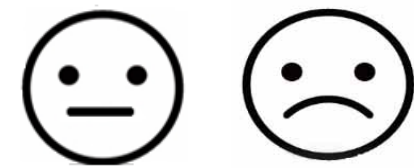

3. Estoy Asustado

(a)

1.¿Sentarte en la sala de espera?

2.¿Un odontólogo lleve un tapabocas (máscara)?

3.¿Acostarte en la silla odontológica?

4. ¿Un odontólogo revise tus dientes con un espejo?

5. ¿Tener un sabor extraño en tu boca, ejemplo calzas*/guantes?

6. ¿Tener una sensación de pinchazo en tu encía?

7. ¿Sentir un entumecimiento en tu labio/lengua?

8. ¿Qué el odontólogo lave tus dientes con un brazo eléctrico que vibra y que salpica agua?

9. ¿Los sonidos que escuchas en el consultorio odontológico?

10. ¿Los olores del consultorio odontológico?

11.¿Qué te saquen un diente?

12. ¿Usar una tela de caucho en tu boca durante el tratamiento?

13. ¿Ser sujetadas las manos o brazos?

\section{B. Parte cognitiva}

Para Niños

14. ¿Te sientes tímido (a) en el consultorio odontológico?

15. ¿Te sientes tímido (a) debido a como se ven sus dientes?

16. ¿Te preocupa perder el control en consultorio odontológico?

Para Padres

17. ¿Su hijo ha tenido tratamientos odontológicos anteriores?

Para Padres

Esté Feliz

Esté bien

18. ¿Cómo espera que su hijo se comporte hoy?
Si

No

Si $\quad$ No

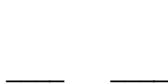

\section{Estoy Bien}
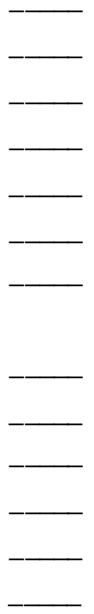

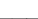


Tabla II. Matriz de configuración (Patrón) de la Escala de Ansiedad Dental Abeer para niños adaptada.

\begin{tabular}{ccccc}
\hline Pregunta & \multicolumn{3}{c}{ Factores } \\
\cline { 2 - 5 } & $\begin{array}{c}\text { Pre- } \\
\text { intervención }\end{array}$ & $\begin{array}{c}\text { Estímulos } \\
\text { restrictivos y/o } \\
\text { invasivos }\end{array}$ & $\begin{array}{c}\text { Estímulos inocuos } \\
\text { (tacto y gusto) }\end{array}$ & $\begin{array}{c}\text { Estímulos inocuos } \\
\text { (olfato y audición) }\end{array}$ \\
\hline EAA3 & $\mathbf{0 , 8 7 8}$ & 0,029 & 0,003 & $-0,040$ \\
EAA2 & $\mathbf{0 , 8 7 5}$ & $-0,041$ & $-0,005$ & $-0,026$ \\
EAA4 & $\mathbf{0 , 8 5 5}$ & 0,019 & 0,045 & $-0,090$ \\
EAA1 & $\mathbf{0 , 6 6 4}$ & $-0,002$ & $-0,313$ & 0,068 \\
EAA12 & $-0,087$ & $\mathbf{0 , 8 1 4}$ & $-0,048$ & $-0,115$ \\
EAA13 & $-0,144$ & $\mathbf{0 , 7 7 8}$ & $-0,276$ & 0,016 \\
EAA11 & 0,190 & $\mathbf{0 , 7 3 2}$ & 0,203 & 0,001 \\
EAA6 & 0,061 & 0,034 & $-\mathbf{0 , 8 7 3}$ & 0,029 \\
EAA7 & 0,042 & $-0,016$ & $-\mathbf{0 , 7 2 0}$ & $-0,280$ \\
EAA5 & 0,309 & 0,141 & $-\mathbf{0 , 6 2 2}$ & $-0,033$ \\
EAA10 & $-0,030$ & 0,026 & 0,129 & $\mathbf{- 0 , 9 0 5}$ \\
EAA8 & 0,017 & 0,025 & $-0,087$ & $\mathbf{- 0 , 7 2 6}$ \\
EAA9 & 0,133 & 0,006 & $-0,172$ & $\mathbf{- 0 , 6 9 9}$ \\
\hline
\end{tabular}

dolor provocado por los demás procedimientos odontológicos. El segundo factor con saturaciones entre 0,814 y 0,732 , se denominó ansiedad ante «estímulos restrictivos y/o invasivos», compuesto por ítems como extraer dientes, usar aislamiento total y ser sujetado de las manos o brazos, que causan mayor dolor o altos niveles de tensión o ansiedad. El tercer factor con saturaciones entre $-0,873$ y $-0,622$, se denominó ansiedad ante «estímulos inocuos de tacto o gusto», en éste se agrupan los ítems que en su naturaleza no generan riesgo para el paciente, pero que están asociados con estímulos propios de los procedimientos odontológicos, tales como la sensación de

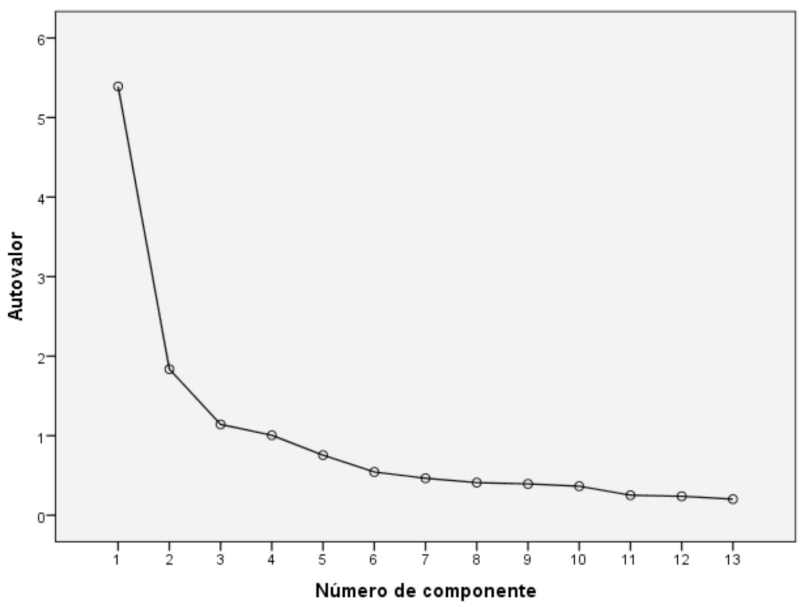

Fig. 2. Sedimentación del Cuestionario Abeer para Ansiedad dental en niños usando análisis factorial exploratorio con rotación oblicua. un pinchazo en las encías, entumecimiento en el labio o lengua y sabores extraños en la boca. El cuarto factor con saturaciones de -0,905 y -0,699, se denominó ansiedad ante "estímulos inocuos de olfato o audición», ya que al igual que el factor anterior no son estímulos propiamente dañinos o dolorosos y que se asocian con los sentidos del tacto y del olfato. En este factor se concentran los ítems relacionados con el sonido de la pieza de baja velocidad, los sonidos del consultorio o los olores que este produce.

Basados en la anterior organización, se calcularon las puntuaciones por sub-escala, con las cuales se elaboró la respectiva matriz de correlaciones, que se presenta en la Tabla III. Se encuentran correlaciones significativas entre todos los componentes, destacándose las altas correlaciones de los estímulos inocuos con los demás factores.

Validez discriminante de la escala. Con el fin de determinar si la escala permite discriminar a los niños de acuerdo con su grado de ansiedad, se solicitó a los "operadores" que valoraran el comportamiento del niño en una escala de 1 a 3, donde 1 era "Feliz", 2 era "Está bien" y 3 era "Asustado". De acuerdo con lo anterior, se puede considerar que la escala discrimina si se encuentran diferencias significativas en las puntuaciones de la escala total y las sub-escalas, según los grupos identificados por los "operadores". En la Tabla IV se presentan las medias y desviaciones estándar de las puntuaciones, según el grupo en el que se ubicaba al niño. Se puede observar cómo en todos los 
Tabla III. Matriz de correlaciones de Spearman entre los componentes de la Escala de Ansiedad Dental Abeer para niños adaptada.

\begin{tabular}{|c|c|c|c|c|}
\hline Factores & Pre-intervención & $\begin{array}{l}\text { Estímulos } \\
\text { restrictivos y/o } \\
\text { invasivos }\end{array}$ & $\begin{array}{l}\text { Estímulos inocuos } \\
\text { (tacto y gusto) }\end{array}$ & $\begin{array}{l}\text { Estímulos inocuos } \\
\text { (olfato y audición) }\end{array}$ \\
\hline Pre-intervención & 1 & & & \\
\hline Estímulos restrictivos y/o invasivos & $0,203^{*}$ & 1 & & \\
\hline Estímulos inocuos (tacto y gusto) & $0,581^{\text {** }}$ & $0,352^{\star *}$ & 1 & \\
\hline Estímulos inocuos (olfato y audición) & $0,476^{\star *}$ & $0,409^{\star *}$ & $0,496^{\star *}$ & 1 \\
\hline
\end{tabular}

casos, a medida que se incrementaba la evaluación del operador, se presentaba un aumento de la calificación en las puntuaciones.

Con el fin de comprobar la hipótesis de trabajo se llevó a cabo un análisis de varianza, en el cual se demostró cómo en todos los casos, había una diferencia estadísticamente significativa $(P<0,001)$ en las puntuaciones total y por sub-escalas, de acuerdo con la valoración dada por el "operador", lo cual demuestra la capacidad del instrumento de discriminar a los niños con alta ansiedad dental (3:Asustado) de los niños con baja ansiedad (1:Feliz) (Tabla IV). Por otro lado, la concordancia de Kappa entre el reporte del operador y el auto-informe del niño de no tener ansie- dad (1:Feliz y 2:Estar Bien) y tener ansiedad (3:Asustado) fue 0,$492 ; \mathrm{P}<0,001$

Fiabilidad de la escala. La consistencia interna de la escala global fue alta, con un $\alpha$ de Cronbach de 0,88. De la misma manera, el factor ansiedad ante la «preintervención» (que incluye los ítems EAA1, EAA2, EAA3 y EAA4) presentó un $\alpha$ de Cronbach de 0,88; el de ansiedad ante "estímulos restrictivos y/o invasivos» (ítems EAA11, EAA12 y EAA13) de 0,71; el de ansiedad ante "estímulos inocuos de tacto o gusto» (ítems EAA5, EAA6 y EAA7) un valor de 0,84 y finalmente el de ansiedad ante «estímulos inocuos de olfato o audición» (ítems EAA8, EAA9 y EAA10) un $\alpha$ de Cronbach de 0,76 .

Tabla IV. Estadística descriptiva en la escala total y las sub-escalas de la Escala de Ansiedad Dental Abeer para niños adaptada, de acuerdo con el nivel evaluado por el operador.

\begin{tabular}{llcccc}
\hline \multicolumn{1}{c}{ Factores } & & $\boldsymbol{n}$ & Media & DE & Valor $\mathbf{P}^{*}$ \\
\hline \multirow{3}{*}{ Pre- intervención } & Feliz & 69 & 4,739 & 1,232 & $<0,001$ \\
& Está bien & 87 & 5,632 & 1,868 & \\
& Asustado & 44 & 6,727 & 2,316 & \\
& Total & 200 & 5,565 & 1,929 & \\
Estímulos restrictivos y/o invasivos & Feliz & 69 & 7,463 & 1,301 & $<0,001$ \\
& Está bien & 87 & 7,574 & 1,244 & \\
& Asustado & 44 & 8,704 & 0,701 & \\
& Total & 200 & 7,785 & 1,263 & \\
Estímulos inocuos (tacto y gusto) & Feliz & 69 & 5,579 & 1,936 & $<0,001$ \\
& Está bien & 87 & 6,885 & 1,195 & \\
& Asustado & 44 & 8,068 & 1,020 & \\
& Total & 200 & 6,695 & 1,728 & \\
& Feliz & 69 & 5,347 & 1,532 & $<0,001$ \\
& Está bien & 87 & 6,195 & 1,453 & \\
Estímulos inocuos (olfato y audición) & Asustado & 44 & 7,386 & 1,104 & \\
& Total & 200 & 6,165 & 1,593 & \\
& Feliz & 69 & 23,130 & 4,297 & $<0,001$ \\
& Está bien & 87 & 26,287 & 4,060 & \\
& Asustado & 44 & 30,886 & 3,724 & \\
Ansiedad Total & Total & 200 & 26,210 & 4,955 & \\
\hline
\end{tabular}

*Derivado de ANOVA 
MAFLA, A. C.; VILLALOBOS, F. H; PINCHAO, R. W. M. \& LUCERO, Y. D. F. Propiedades psicométricas de la versión española de la Abeer Children Dental Anxiety Scale (ACDAS) para la medición de ansiedad dental en niños. Int. J. Odontostomat., 11(2):182-191, 2017.

Puntuación de la Escala. La puntuación media de la escala total fue $26,21(\mathrm{DE}=4,955)$ y, dentro de los factores, el grupo de estímulos restrictivos y/o invasivos tuvo una mayor media $(x=7,785$; $D E=1,263)$, seguida por los inocuos por tacto y gusto $(x=6,695 ; D E=1,728)$ y por olfato $y^{-}$audición $(x=6,165, D E=1,593)$ y mucho menor los de pre-intervención $(x=5,565, D E=1,929)$. Según valores $Z$, se determinó una prevalencia de 34 (17\%) que tuvieron ansiedad dental. Existieron diferencias significativas por edad, en niños de 5 años se encontra- ron 5 casos de ansiedad (62,5\%), en los de 6 años, 9 casos (18,8 \%); en los de 7 años, 12 casos $(22,6$ $\%)$; en los de 8 años, 5 casos (11,6\%); en los de 9 años, 3 casos ( $9,7 \%)$; y en los de 10 años, ninguno $(\mathrm{P}=0,002)$, lo que demuestra un decremento gradual de la ansiedad con la edad. No existieron diferencias según sexo en la prevalencia de ansiedad dental. Por otro lado, se encontró que no existieron diferencias significativas en las puntuaciones según las sub-escalas obtenidas según el sexo, pero sí por grupos de edades (Tabla V).

Tabla V. Escala total y sub-escalas de la Escala de Ansiedad Dental Abeer para niños adaptada, de acuerdo con edad.

\begin{tabular}{|c|c|c|c|c|c|}
\hline Factores & $\begin{array}{l}\text { Edad } \\
\text { (años) }\end{array}$ & $\mathrm{n}$ & Media & $\mathrm{DE}$ & Valor $\mathrm{P}^{*}$ \\
\hline & 5 & 8 & 7,750 & 2,866 & 0,004 \\
\hline & 6 & 48 & 5,645 & 2,138 & \\
\hline & 7 & 53 & 5,754 & 1,764 & \\
\hline \multirow[t]{7}{*}{ Pre - intervención } & 8 & 43 & 5,441 & 1,790 & \\
\hline & 9 & 31 & 5,322 & 1,720 & \\
\hline & 10 & 17 & 4,470 & 1,124 & \\
\hline & Total & 200 & 5,565 & 1,929 & \\
\hline & 5 & 8 & 8,500 & 0,755 & 0,011 \\
\hline & 6 & 48 & 7,750 & 1,376 & \\
\hline & 7 & 53 & 8,037 & 1,073 & \\
\hline \multirow[t]{7}{*}{ Estímulos restrictivos y/o invasivos } & 8 & 43 & 7,465 & 1,297 & \\
\hline & 9 & 31 & 8,064 & 1,152 & \\
\hline & 10 & 17 & 7,058 & 1,390 & \\
\hline & Total & 200 & 7,785 & 1,263 & \\
\hline & 5 & 8 & 8,125 & 1,125 & 0,020 \\
\hline & 6 & 48 & 6,854 & 1,688 & \\
\hline & 7 & 53 & 6,679 & 1,939 & \\
\hline \multirow[t]{7}{*}{ Estímulos inocuos (tacto y gusto) } & 8 & 43 & 6,511 & 1,517 & \\
\hline & 9 & 31 & 6,935 & 1,526 & \\
\hline & 10 & 17 & 5,647 & 1,765 & \\
\hline & Total & 200 & 6,695 & 1,728 & \\
\hline & 5 & 8 & 7,500 & 0,925 & 0,005 \\
\hline & 6 & 48 & 6,250 & 1,550 & \\
\hline & 7 & 53 & 6,301 & 1,682 & \\
\hline \multirow[t]{7}{*}{ Estímulos inoquos (olfato y audición) } & 8 & 43 & 6,070 & 1,386 & \\
\hline & 9 & 31 & 6,258 & 1,569 & \\
\hline & 10 & 17 & 4,941 & 1,638 & \\
\hline & Total & 200 & 6,165 & 1,593 & \\
\hline & 5 & 8 & 31,875 & 4,673 & $<0,001$ \\
\hline & 6 & 48 & 26,500 & 5,488 & \\
\hline & 7 & 53 & 26,773 & 4,967 & \\
\hline \multirow[t]{4}{*}{ Ansiedad Total } & 8 & 43 & 25,488 & 4,031 & \\
\hline & 9 & 31 & 26,580 & 4,248 & \\
\hline & 10 & 17 & 22,117 & 3,722 & \\
\hline & Total & 200 & 26,210 & 4,955 & \\
\hline
\end{tabular}

*Derivado de ANOVA. 


\section{DISCUSIÓN}

La ansiedad dental en niños puede estar relacionada con múltiples estímulos, como agujas, extracciones dentales, sonido de las piezas de mano, percepción negativa del odontólogo frente a la salud oral del paciente, entre otras. De acuerdo a las propiedades psicométricas de la escala Abeer, encontramos que el análisis factorial exploratorio evidencia la existencia de un modelo teóricamente coherente para la evaluación de ansiedad dental. El valor de $\alpha$ de Cronbach de 0,88 indicó una alta fiabilidad general del instrumento. En el análisis se observó que la Escala Abeer tiene buenas características estructurales que determinaron cuatro sub-escalas con buena consistencia interna. Estas escalas se denominaron ansiedad ante la «pre-intervención» con un $\alpha$ de Chronbach de 0,88; ansiedad ante "estímulos restrictivos y/o invasivos» de 0,71 ; ansiedad ante "estímulos inocuos de tacto o gusto» de 0,84 y ansiedad ante «estímulos inocuos de olfato o audición)» de 0,76 , lo que indica que son confiables para ser utilizadas en la medición de ansiedad dental en estos niños. Debido a que no se cuenta con otros estudios que utilicen este mismo instrumento, y tampoco con el ajuste realizado, la comparación de estos datos psicométricos se ve limitada.

La agrupación de cuatro sub-escalas y su respectiva denominación, es importante para el manejo de la ansiedad dental. Por ejemplo, la primera relacionada con los estímulos propios de la «pre-intervención», se puede decir que a diferencia de la cita médica convencional, donde el paciente por lo general comenta sus síntomas, en una cita odontológica en la mayoría de los casos se espera que se realice un procedimiento, ya que por lo general los pacientes no acuden para revisiones generales preventivas sino por cuadros que incluyen posiblemente signos y síntomas como dolor, inflamación o sangrado. En el caso de los niños, las primeras revisiones se realizan de acuerdo al interés y conocimientos sobre salud oral de los padres. Al respecto, una condición socio-económica baja se relacionó con desconocimiento sobre temas de salud oral en mujeres embarazadas (Vilella et al., 2016), lo que podría influir sobre las visitas preventivas odontológicas que sus hijos deberían hacer. La ansiedad pre-quirúrgica es caracterizada por sentimientos subjetivos de tensión, aprensión, nerviosismo y preocupación. Esta ansiedad se relaciona con miedos a que el tratamiento sea fallido, pérdida de control o muerte (de Groot, et al., 1997). Esta ansiedad se presenta en cualquier persona, puede ser de forma transitoria o crónica, y también puede producir reacciones agresivas en los pacientes que aumentan el estrés experimentado por ellos, causando un manejo del dolor mucho más difícil que el del posoperatorio (Anderson \& Masur, 1983). En odontología, conductas como gritar, dar patadas en la silla odontológica, cerrar la boca con fuerza o morder al odontólogo en las manos, en la fase de pre-intervención se evidencian con relativa frecuencia.

En relación con la dimensión «estímulos irestrictivos y/o invasivos», son condiciones que ocasionan dolor, por ejemplo, la Odontología involucra diferentes tipos de procedimientos, los cuales podrían ser percibidos como molestos por los niños, entre ellos las extracciones dentales siendo una de las más dolorosas que puede ocasionar distrés psicológico (Klingberg et al., 1994). Por otra parte, mecanismos que aunque protegen a los niños de lesiones durante el tratamiento odontológico como el uso de la tela de caucho y el sujetar las manos o brazos, podrían ocasionar distrés psicológico y desarrollar fobia a la odontología (Weaver, 2010). Por esta razón, Clinical Affairs Committee-Behavior Management Subcommittee \& American Academy of Pediatric Dentistry (2015) sugieren este tipo de restricción cuando el paciente requiere un diagnóstico inmediato y / o tratamiento limitado y no puede cooperar debido a la falta de madurez o discapacidad mental o física; cuando la seguridad del paciente, el personal, el odontólogo, o padre estaría en riesgo sin restricciones y cuando el paciente está sedado y requiere una estabilización limitada para ayudar a disminuir el movimiento inapropiado.

Las dimensiones de "estímulos inocuos» (tacto-gusto y olfato-audición)», son condiciones que se perciben por diferentes sentidos, y tienen menor intensidad que las anteriores, sin embargo, pueden ser muy molestas o menos molestas para ciertas personas. Por ejemplo, es el caso de sentir un pinchazo en la encía (Vika et al., 2008) o sentirse inquieto por los sonidos u olores del consultorio. Lalic et al. (2015), en niños serbios de 12 años, evidenciaron que el mayor temor en la consulta odontológica es el tratamiento con la pieza de alta velocidad (perforación), por ejemplo. Estas situaciones pueden coincidir con la teoría de Iván Pavlov (1927), sobre condicionamiento clásico, según la cual un individuo forma una asociación entre dos eventos. Lo más importante es que uno predice el otro, por ejemplo, el sonido de la pieza de alta 
velocidad se puede asociar con el dolor qué esta ocasiona cuando los dientes son perforados, lo cual es memorizado por los niños. Una vez ellos regresan a la atención odontológica, el sonido de la pieza de alta se relaciona con dolor, lo cual es suficiente para producir ansiedad, la que podría ser manifestada con tensión muscular, sudoración en las manos y lloriqueo. También podría manifestarse, cuando el paciente lo recuerda aún sin que el estímulo esté presente, como al preguntarlo en esta encuesta.

Esta escala fue aplicada a niños que ya tenían experiencias previas, los resultados estadísticos nos muestran que es un buen instrumento para discriminar los niveles de baja y alta ansiedad referidos por los niños y el operador. El índice de Kappa fue 0,492; $\mathrm{P}<0,001$ con un resultado similar al encontrado por Buchanan \& Niven (2003) en el Facial Image Scale $(0,45 ; \mathrm{P}<0,05)$.

La prevalencia de ansiedad dental estimada con esta escala fue de $17 \%$, resultado similar al encontrado por Soares et al. (2016) en niños entre 5-7 años con la escala Dental Anxiety Question (DAQ) o por Popescu et al. en niños de 6 a 12 años que fue 22,68 $\%$ con la Dental Anxiety Scale (DAS).Es importante mencionar que la ansiedad dental disminuyó con la edad en este estudio, resultados similares fueron observados por Raja et al. (2015) en niños de 5 a10 años de Pakistán, por Mishra et al. (2016), en niños de 3-14 años de la India; y Popescu et al. en niños escolares de Rumania.

La ansiedad dental es un problema importante en niños, porque puede afectar la salud oral del paciente debido a comportamientos de evitación de tratamientos como resultado de este sentimiento. Por esta razón, el uso de herramientas para su detección es importante en el manejo de los niños que asisten a la consulta odontológica. La Escala Abeer, es una excelente herramienta para este fin, ya que sus propiedades psicométricas como validez interna, capacidad de discriminación y fiabilidad son buenas y es de fácil aplicación.

\section{AGRADECIMIENTOS}

El grupo investigador agradece a los niños que colaboraron con el diligenciamiento de la escala, así como a los padres que autorizaron que sus hijos que asisten a la Clínica Odontológica de la Universidad
Cooperativa de Colombia de Pasto, participaran en el estudio.

MAFLA, A. C.; VILLALOBOS, F. H; PINCHAO, R. W. M. \& LUCERO, Y. D. F. Psychometric properties of the spanish version of the Abeer Children Dental Anxiety Scale (ACDAS) to dental anxiety in children. Int. J. Odontostomat., 11(2):172181, 2017.

ABSTRACT: The validity and reliability process of dental anxiety scales for children in Spanish has been scarce. The aim of this research was to determine the psychometric properties of a Spanish adaptation of the Abeer Children Dental Anxiety Scale (ACDAS) as a self-report measure of dental anxiety in children. The ACDAS includes 13 items about the anxiety reactions of receiving dental care and a cognitive component assessed to children, parents and operators. We adapted the Abeer Children Dental Anxiety Scale to Spanish language, and then determined its psychometric properties in a sample of 200 children patients from the Dental Clinic at Universidad Cooperativa de Colombia, Pasto, Colombia. The scale's structural validity was estimated through an Exploratory Factor Analysis (EFA) and the reliability was established by examining its internal consistency (Cronbach's $\alpha$ ). The consecutive sample was comprised of 112 men and 88 women and their ages ranged from 5 to 10 years. The EFA loaded a four-factor structure which accounted for $72.12 \%$ of the variance. We labeled to those factors according to anxiety reactions to potential harmful stimuli as "pre-intervention stimuli", "invasive/ restrictive stimuli", "innocuous stimuli (touch, taste)" and innocuous stimuli (smell, hearing)". The internal consistencies of the sub-scales were $0.88,0.71,0.84$ and 0.76 , respectively. The sub-scales showed good convergent, discriminant and construct validity. The results suggest the ACDAS is a valid, reliable, and clinically useful instrument for assessing dental anxiety in children.

KEY WORDS: dental anxiety, cognition, children, survey and questionnaires, psychometrics.

\section{REFERENCIAS BIBLIOGRÁFICAS}

Al-Namankany, A.; Ashley, P. \& Petrie, A. The development of a dental anxiety scale with a cognitive component for children and adolescents. Pediatr. Dent., 34(7):e219-24, 2012a.

Al-Namankany, A.; de Souza, M. \& Ashley, P. Evidence-based dentistry: analysis of dental anxiety scales for children. Br. Dent. J., 212(5):219-22, 2012b.

Anderson, K. O. \& Masur, F. T. 3rd. Psychological preparation for invasive medical and dental procedures. J. Behav. Med., 6(1):140, 1983.

Arapostathis, K. N.; Coolidge, T.; Emmanouil, D. \& Kotsanos, N. Reliability and validity of the Greek version of the children's fear survey schedule-dental subscale. Int. J. Paediatr. Dent., 18(5):374-9, 2008 
MAFLA, A. C.; VILLALOBOS, F. H; PINCHAO, R. W. M. \& LUCERO, Y. D. F. Propiedades psicométricas de la versión española de la Abeer Children Dental Anxiety Scale (ACDAS) para la medición de ansiedad dental en niños. Int. J. Odontostomat., 11(2):182-191, 2017.

Buchanan, H. \& Niven, N. Further evidence for the validity of the Facial Image Scale. Int. J. Paediatr. Dent., 13(5):368-9, 2003.

Buchanan, H. \& Niven, N. Validation of a Facial Image Scale to assess child dental anxiety. Int. J. Paedriatr. Dent., 12(1):47-52, 2002.

Clinical Affairs Committee-Behavior Management Subcommittee \& American Academy of Pediatric Dentistry. Guideline on Behavior Guidance for the Pediatric Dental Patient. Pediatr. Dent., 37(5):57-70, 2015.

Cuthbert, M. I. \& Melamed, B. G. A screening device: children at risk for dental fears and management problems. A. S. D. C. J. Dent. Child., 49(6):432-6, 1982.

de Groot, K. I.; Boeke, S.; van den Berge, H. J.; Duivenvoorden, H. J.; Bonke, B. \& Passchier, J. The influence of psychological variables on postoperative anxiety and physical complaints in patients undergoing lumbar surgery. Pain, 69(1-2):19-25, 1997.

de Menezes Abreu, D. M.; Leal, S. C.; Mulder, J. \& Frencken, J. E. Patterns of dental anxiety in children after sequential dental visits. Eur. Arch. Paediatr. Dent., 12(6):298-302, 2011.

Klingberg, G.; Berggren, U. \& Norén, J. G. Dental fear in an urban Swedish child population: prevalence and concomitant factors. Community Dent. Health., 11(4):208-14, 1994.

Lalic, M.; Aleksic, E.; Milic, J.; Malesevic, A. \& Jovicic, B. Reliability and validity of the Serbian version of Children's Dental Fear Questionnaire. Vojnosanit. Pregl., 72(7):602-7, 2015.

Majstorovic, M.; Veerkamp, J. S. \& Skrinjaric, I. Reliability and validity of measures used in assessing dental anxiety in 5- to 15-year-old Croatian children. Eur. J. Paediatr. Dent., 4(4):197202, 2003.

Mishra, G.; Thakur, S.; Singhal, P.; Ghosh, S. N.; Chauhan, D. \& Jayam, C. Assessment of child behavior in dental operatory in relation to sociodemographic factors, general anxiety, body mass index and role of multi media distraction. J. Indian Soc. Pedod. Prev. Dent., 34(2):159-64, 2016.

Norris, W. \& Baird, W. L. Pre-operative anxiety: a study of the incidence and aetiology. Br. J. Anaesth., 39(6):503-9, 1967.

Paryab, M. \& Hosseinbor, M. Dental anxiety and behavioral problems: a study of prevalence and related factors among a group of Iranian children aged 6-12. J. Indian Soc. Pedod. Prev. Dent., 31(2):826, 2013.

Pavlov, I. P. Conditioned Reflexes (Translated by G. V. Arpeg). London, Oxford University Press, 1927.

Popescu, S. M.; Dascalu, I. T.; Scrieciu, M.; Mercut, V.; Moraru, I. \& Tuculina, M. J. Dental anxiety and its association with behavioral factors in children. Curr. Health Sci. J., 40(4):261-4, 2014.

Raja, G. H.; Malik, F. S.; Bashir, U. \& Attaullah, S. Dental anxiety among children of age between 5 to 10 years visiting a teaching dental hospital in Islamabad, Pakistan. J. Ayub. Med. Coll. Abbottabad, 27(3):587-90, 2015.

Soares, F. C.; Lima, R. A.; Santos, C. da F.; de Barros, M. V. \& Colares, V. Predictors of dental anxiety in Brazilian 5-7 years old children. Compr. Psychiatri., 67:46-53, 2016.

Venham, L. L. \& Gaulin-Kremer, E. A self-report measure of situational anxiety for young children. Pediatr. Dent., 1(2):91-6, 1979.

Venham, L. L. The effect of mother's presence of child's response to dental treatment. A. S. D. C. J. Dent. Child., 46(3):219-25, 1979.

Vika, M.; Skaret, E.; Raadal, M.; Ost, L. G. \& Kvale, G. Fear of blood, injury, and injections, and its relationship to dental anxiety and probability of avoiding dental treatment among 18-year-olds in Norway. Int. J. Paediatr. Dent., 18(3):163-9, 2008.

Vilella, K. D.; Alves, S. G.; de Souza, J. F.; Fraiz, F. C. \& Assunção, L. R. The Association of Oral Health Literacy and Oral Health Knowledge with Social Determinants in Pregnant Brazilian Women. J. Community Health, 41(5):1027-32, 2016.

Weaver, J. M. Why is physical restraint still acceptable for dentistry? Anesth. Prog., 57(2):43-4, 2010.

\author{
Dirección para correspondencia: \\ Ana Cristina Mafla \\ Facultad de Odontología \\ Universidad Cooperativa de Colombia \\ Calle 18 No. 47 - 150 \\ Pasto, Nariño \\ COLOMBIA
}

E-mail: ana.mafla@campusucc.edu.co

Recibido : 03-11-2016

Aceptado: 05-05-2017 